\title{
Evaluation of an Intravenous-Endovascular Strategy in Patients with Acute Proximal Middle Cerebral Artery Occlusion
}

\author{
J.-F. Vendrell, R. Mernes, N. Nagot, D. Milhaud, K. Lobotesis, V. Costalat, P. Machi, I.L. Maldonado, \\ C. Riquelme, C. Arquizan, and A. Bonafe
}

\begin{abstract}
BACKGROUND AND PURPOSE: IVT administered in acute ischemic stroke provides low recanalization rates in proximal intracranial occlusions, with consequently poor clinical outcome. The safety and efficacy of an IES by using mechanical thrombectomy after IVT failure were assessed in acute MCA occlusions.

MATERIALS AND METHODS: Patients presenting with acute MCA occlusion within 4.5 hours with an NIHSS score between 8 and 25 and a DWI ASPECTS of $>5$ were eligible. From September 2009 to September 2010, mechanical thrombectomy by using the Solitaire FR device was systematically performed if no clinical improvement was observed 1 hour after the initiation of IVT (IES group). Results in terms of clinical outcome were compared with those from an IVT series from January 2007 to August 2009 (IVT group).

RESULTS: Alteplase was administered in 123 patients with proximal intracranial occlusion. Fifty-six had a confirmed MCA occlusion: 32 were included in the IVT group; and 24, in the IES group. At 24 hours, the median NIHSS improvement was 8.5 points in the IES group $(25 \%-75 \% \mathrm{Cl}, 1.5-13)$ and 3 points in the IVT group $(25 \%-75 \% \mathrm{Cl}, 1-5)(P=.001)$. At 3 months, $17 / 22(77 \%)$ patients from the IES group and $15 / 30$ (50\%) from the IVT group had an $\mathrm{mRS}$ score of $\leq 2$. After adjustment for confounding variables, IES was strongly associated with favorable clinical outcome $(77 \%$ versus $50 \%$; adjusted odds ratio $=11.9 ; 95 \% \mathrm{Cl}, 1.6-89.1 ; P<.02)$. No symptomatic intracranial hemorrhage was observed.
\end{abstract}

CONCLUSIONS: IES by using systematic mechanical thrombectomy after IVT failure safely improves the clinical outcome at 3 months and could represent an interesting alternative in the management of patients with acute MCA occlusion.

ABBREVIATIONS: ASPECTS = Alberta Stroke Program Early CT Score; CI Score = confidence interval; IA = intra-arterial; IES = IV-endovascular strategy; IVT = IV thrombolysis; $\mathrm{mRS}$ = modified Rankin Scale

I

V recombinant tissue plasminogen activator therapy is the standard treatment for acute ischemic stroke within 4.5 hours of symptom onset. ${ }^{1}$ However, this therapeutic approach remains unsatisfactory with poor recanalization rates for proximal intracranial occlusions (32\%-35\%), though more favorable outcomes are reported for the MCA. ${ }^{2,3}$ Given the strong correlation between prompt recanalization and favorable clinical outcome, more invasive approaches such as IA thrombolysis, bridging IV-IA rescue strategy, and combined IV-IA thrombolysis have been recommended (recanalization rates ranging from $45 \%$ to $71 \%){ }^{4-8}$ In

Received December 19, 2011; accepted after revision May 14, 2012.

From the Departments of Neuroradiology (J.-F.V., K.L., V.C., P.M., I.L.M., C.R., A.B.), Neurology (R.M., D.M., C.A.), and Medical Informatics (N.N.), Montpellier University Hospital, Montpellier, France.

This work was supported by the University Hospital Center of Montpellier, France.

Please address correspondence to Jean-François Vendrell, MD, Department of Neuroradiology, Montpellier University Hospital Center, 80 Ave Augustin Fliche 34295 Montpellier Cedex 5. France; e-mail: jfvendrell@yahoo.fr

http://dx.doi.org/10.3174/ajnr.A3230 cases of persistent proximal artery occlusion following IV or IA thrombolysis, additional mechanical thrombectomy was proposed with promising results in terms of recanalization (87\%) compared with IVT alone (52\%). Clinical outcome tended to be better at 3 months following thrombectomy ( $\mathrm{mRS} \leq 2$ in $57 \%$ of patients) but without reaching statistical significance $(P=.35)^{9}{ }^{9}$

Regarding the MCA topography, limited data following an IV thrombectomy strategy are available to date in terms of recanalization rate $(74 \%-88 \%)$ and favorable patient clinical outcome at 3 months (41\%-63\%). ${ }^{10,11}$ Although these results have to be confirmed, they may be tempered by complications associated with the technique (ie, intracranial hemorrhage). In this respect and to avoid unnecessary thrombectomies in patients who would potentially have a successful IVT, we designed an IES, which systematically included an IVT for all patients and then a thrombectomy as a second-line therapy in cases of IVT failure. The aim of this study was to assess the safety and efficacy of an IES in comparison with an historical standard IVT series for patients presenting with an 
acute ischemic stroke due to an MCA occlusion within 4.5 hours of onset.

\section{MATERIALS AND METHODS \\ Patients}

From January 2007 to September 2010, all consecutive patients presenting with an acute ischemic stroke at Montpellier University Hospital, France, were included in this work if they fulfilled the following criteria: 1) a symptom onset from IVT infusion of $<4.5$ hours, 2) a stroke in the MCA territory with a confirmed proximal occlusion (M1 segment) on MRA, and 3) an NIHSS score between 8 and 25. Patients who presented with at least 1 of the following criteria were excluded from the study: 1) a thrombolysis contraindication either in accordance with the National Institute of Neurological Disorders and Stroke guidelines ${ }^{12}$ or a derived MR imaging ASPECTS of $<5$, indicating an extensive stroke; and 2) an associated occlusion in another intracranial arterial territory or a tandem occlusion in the internal carotid artery.

\section{Study Design}

As a routine practice, all patients presenting with an acute stroke underwent MRA a few minutes after their admission in parallel with an initial NIHSS score assessment. Pacemaker and patient agitation were the main MRA contraindications.

After a review of our therapeutic strategy in September 2009, ${ }^{13}$ eligible patients were then split into 2 groups: 1) Patients from the first study period (January 2007 to August 2009, 32 months) treated with standard IVT alone were registered in the IVT group, and 2) patients from the second study period (September 2009 to September 2010, 12 months) treated by using the IES were registered in the IES group. The IES group included patients who received either a rescue thrombectomy following IVT failure or a successful IVT. Demographic data, medical history, vascular risk factors, imaging findings, and clinical data for every patient with a confirmed acute ischemic stroke were retrieved from the medical records. The clinical outcome of patients was systematically assessed by a neurologist by using the NIHSS during the acute phase (at admission and 24 hours later) and the mRS at 3 months. An mRS score ranging from 0 to 2 was considered a favorable clinical result. ${ }^{9}$ Informed consent was obtained from all patients or family members, and the local ethics committee approved the study.

\section{Procedures}

In our institution, all patients presenting with a suspected acute ischemic stroke had systematic MRA on admission. DWI, T2, FLAIR, 3D time-of-flight sequences, and neck vessels were acquired and analyzed to confirm the ischemic lesion and the MCA occlusion. As described, the ASPECTS was applied to the DWI sequence to evaluate the parenchymal extension of the ischemic lesion. ${ }^{14}$ Once the decision to treat was made, IVT was immediately administered. In the first study period, alteplase alone (0.9 $\mathrm{mg} / \mathrm{Kg}$ ) was infused with systematic clinical examinations (NIHSS score) at 1 hour and 24 hours. In the second study period, additional mechanical thrombectomy was discussed for each patient after the 1-hour NIHSS score. If this second NIHSS score was $<8$ or if it improved by at least 4 points in comparison with the baseline score, IVT was considered successful without additional therapy.

In cases of failed IVT, a mechanical thrombectomy was undertaken as soon as possible, by using a stent retriever (Solitaire FR, $4 \times 20 \mathrm{~mm}$; ev3, Irvine, California), with the patient under general anesthesia. An 8F Merci balloon-guiding catheter (Concentric Medical, Mountain View, California) was introduced through a femoral sheath into the appropriate carotid artery. A 0.021inch-internal-diameter microcatheter, Prowler Select Plus (Cordis, Miami Lakes, Florida), was navigated distal to the point of occlusion over an 0.014-inch steerable microwire. A microcatheter angiographic run was then carried out to confirm the microcatheter position distal to the thrombus. The Solitaire FR was then introduced through the microcatheter, and the device was deployed across the occluding thrombus. The microcatheter distal marker was positioned at the exact level of the proximal marker of the stent. An intravenous bolus of 1000 IU of standard heparin was administered at this time. On Solitaire FR deployment, an angiographic control was performed to evaluate the correct placement and expansion of the device. After the angiographic run, the Solitaire FR was maintained in place for a few minutes (3-7 minutes) to allow device expansion. After this time, the fully deployed Solitaire FR and the delivery microcatheter were gently pulled back together and recovered through the guiding catheter. The Solitaire FR was at no point completely or partially resheathed. During the retrieval, the balloon-guide catheter was inflated to suspend anterograde flow.

Manual aspiration with a $50-\mathrm{mL}$ syringe was performed through the hemostatic valve during the retrieval to reverse the flow and to aspirate clot debris eventually present in the lumen of the guide catheter. A maximum of 5 passes was performed by using the device, if necessary. The integrity of the stent was consistently checked after every pass. No additional devices were used with the Solitaire FR, and it was not used in combination with intra-arterial rtPA in any case. The final MCA recanalization rate was assessed on digital subtraction angiography by using the Thrombolysis In Cerebral Infarction scoring system. ${ }^{15}$ A noncontrast CT was performed at day 1 postprocedure in all patients to exclude intracranial hemorrhage. Intracranial hemorrhage was considered symptomatic if a related increase of the NIHSS score of $>4$ points was observed. The Solitaire FR device is certified by the European Union.

\section{Statistical Analysis}

The main characteristics of patients were described at baseline to identify any significant clinical difference between the 2 groups. The Fisher exact and $t$ tests were used to compare qualitative and quantitative variables between the 2 groups, respectively. The NIHSS score was also compared at 24 hours. At 3 months, an mRS score of $\leq 2$ was used to ascertain a good clinical outcome. Association between IES and favorable outcome at 3 months was assessed by using an exact logistic regression analysis, adjusting for the initial NIHSS score, the most important confounding factor in our study. Due to the relatively small number of events in our sample, a regular logistic regression model would not be appropriate because the underlying asymptotic theory would not apply. 


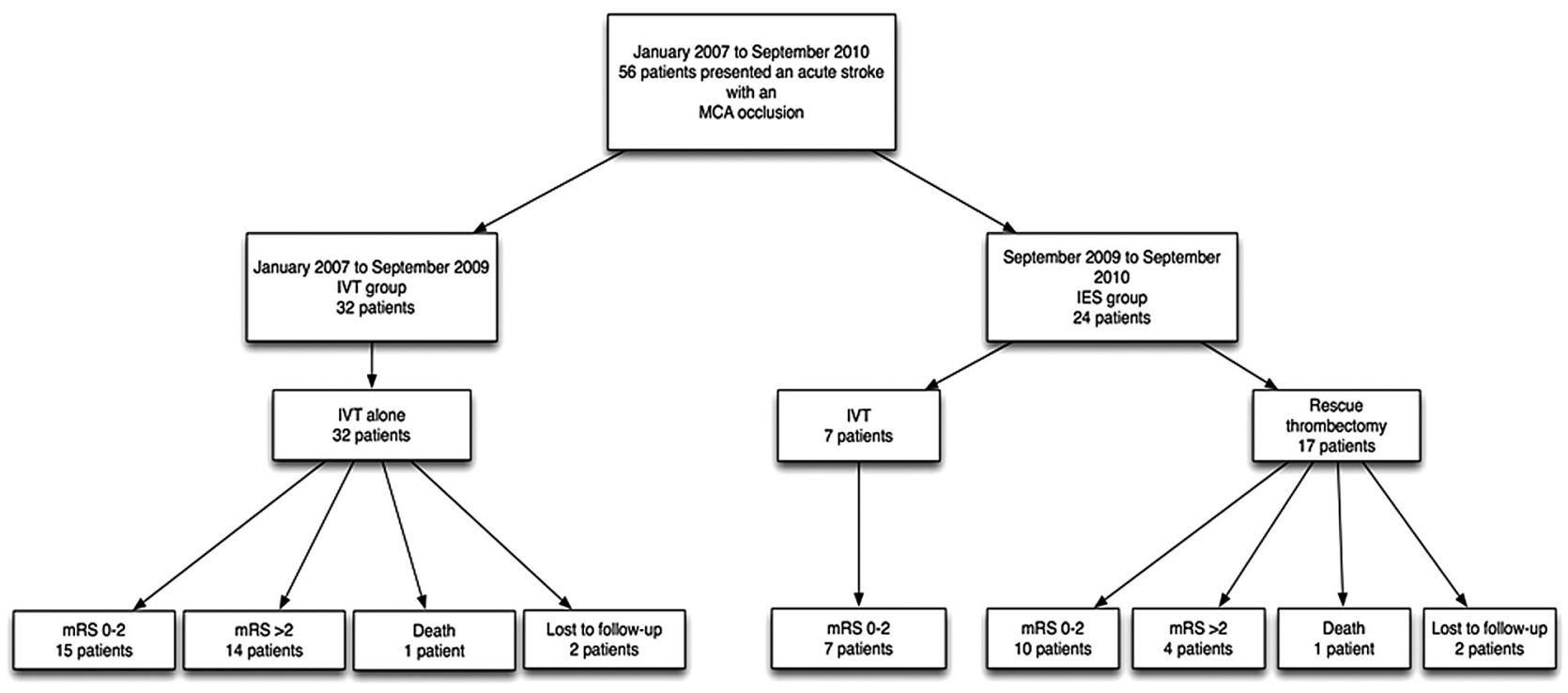

FIG 1. Flowchart of patients and outcome at 3 months.

Table 1: Patient characteristics before treatment

\begin{tabular}{lccc}
\hline & $\begin{array}{c}\text { IVT } \\
(\boldsymbol{n}=\mathbf{3 2})\end{array}$ & $\begin{array}{c}\text { IES } \\
(\boldsymbol{n}=\mathbf{2 4})\end{array}$ & $\begin{array}{c}\boldsymbol{P} \\
\text { Value }^{\mathrm{a}}\end{array}$ \\
\hline Age (yr, SD) & $71.5(11.7)$ & $69.0(12.0)$ & .44 \\
Women & $16(50.0 \%)$ & $8(33.3 \%)$ & .21 \\
Medical history & & & \\
$\quad$ Hypertension & $22(68.7 \%)$ & $12(50.0 \%)$ & .16 \\
$\quad$ Hypercholesterolemia & $15(46.9 \%)$ & $4(16.7 \%)$ & .02 \\
$\quad$ Diabetes & $5(15.6 \%)$ & $5(20.8 \%)$ & .73 \\
$\quad$ Current smoker & $12(37.5 \%)$ & $8(33.3 \%)$ & .75 \\
$\quad$ Cardiovascular disease & $17(53.1 \%)$ & $10(41.7 \%)$ & .40 \\
Clinical data & & & \\
$\quad$ Initial NIHSS score & $13.3(6.3)$ & $15.2(5.5)$ & .25 \\
Time from onset to IVT (min) & $195(46.4)$ & $169(50.7)$ & .046 \\
Door-to-needle time (min) & $69 \pm 12$ & $62 \pm 13$ & .2 \\
\hline
\end{tabular}

${ }^{a}$ For comparison between IVT and IES (Fisher exact test or $t$ test for continuous variables).

All statistical analyses were carried out by using STATA, Version 11 software (StataCorp, College Station, Texas).

\section{RESULTS}

\section{Patient and Procedure Characteristics}

From January 2007 to September 2010, a total of 256 patients received IVT for an acute ischemic stroke within 4.5 hours of symptom onset. Among them, 123 (48\%) presented with a confirmed proximal artery occlusion, including 56 (22\%) within the M1 segment. Thirty-two of 56 (57\%) were in the IVT group, and $24 / 56(43 \%)$ were in the IES group (Fig 1). Mean age, sex ratio, medical history, clinical score, and time from the onset of symptoms to IVT are summarized in Table 1. Overall, the patient characteristics were similar between the 2 groups. However, patients included in the IVT group tended to have a better initial NIHSS score than those in the IES group (median NIHSS $=15$ [25\%75\% CI, 7-19] and 16 [25\%-75\% CI, 12-19], respectively) and a higher mean time from symptom onset $(195 \pm 46.4$ versus $169 \pm$ 50.7 minutes, respectively). These were not shown to be significant (Table 1), and an adjustment for confounding variables between the 2 groups was performed for the interpretation of our results. The mean door-to-needle time was $62 \pm 13$ minutes in the IES group and $69 \pm 12$ minutes in the IVT group (Table 1). The IES group included 7/24 (29\%) cases of successful IVT and 17/24 (71\%) cases requiring additional mechanical thrombectomy secondary to IVT failure (Fig 1).

All 56 included patients underwent MRA with a sufficient image quality to make the decision to treat. Four (7\%) were lost to follow-up ( 2 patients in each group) and did not have clinical assessment at 3 months. Two of $56(3.6 \%)$ patients died from malignant ischemic stroke (1 patient in the IVT group and 1 patient in the IES group) and were both considered as having unfavorable results at 3 months $(\mathrm{mRS}=6)$.

\section{Recanalization}

A noncontrast CT was performed at 24 hours in all patients to exclude an intracranial hemorrhage. However, recanalization of the MCA was not systematically assessed after alteplase infusion. In the IES group, all patients undergoing thrombectomy had a persistent M1 occlusion confirmed on initial digital subtraction angiography. Arteries of 16 of 17 patients (94\%) recanalized after thrombectomy with 15/17 (88\%) achieving complete recanalization (Thrombolysis In Cerebral Infarction 3) and 1/17 (6\%) achieving partial recanalization (Thrombolysis In Cerebral Infarction 2a). In 1 case, the MCA did not reconstitute following mechanical thrombectomy (6\%) (Thrombolysis In Cerebral Infarction 0 ). In the 16 patients who benefited from a MCA recanalization, the mean time from symptom onset to recanalization was $297 \pm 61.6$ minutes and the mean time from femoral artery puncture to recanalization was $38 \pm 20.9$ minutes. The mean time from femoral puncture to deployment of the Solitaire device was $26.5 \pm 7.6$ minutes.

\section{Clinical Outcome}

The median NIHSS score improvement between admission and 24 hours was $3(25 \%-75 \% \mathrm{CI}, 1-5)$ points in the IVT group and $8.5(25 \%-75 \% \mathrm{CI}, 1.5-13)$ points in the IES group (difference, 5.5 points; $P<.001$ ) (Table 2). For 6 of 56 participants $(11 \%)$, it was 
Table 2: Initial and midterm results

\begin{tabular}{cccc}
\hline & $\begin{array}{c}\text { IVT } \\
(\boldsymbol{n}=\mathbf{3 0})\end{array}$ & $\begin{array}{c}\text { IES } \\
(\boldsymbol{n}=\mathbf{2 2})\end{array}$ & $\begin{array}{c}\boldsymbol{P} \\
\text { Value }^{\mathrm{a}}\end{array}$ \\
\hline Initial treatment efficacy (24 hr) & & & \\
Mean NIHSS difference at & $-2.8(2.7)$ & $-8.0(7.5)$ & .001 \\
24 hrs from baseline (SD) & & & \\
NIHSS improvement $>4$ & $10(35.7 \%)$ & $15(71.4 \%)$ & .01 \\
NIHSS $=0$ & $4(33.3 \%)$ & $8(66.7 \%)$ & .05 \\
Midterm treatment efficacy & & & \\
$\quad(3$ months) & & & \\
mRS $\leq 2$ & $15(50.0 \%)$ & $17(77.3 \%)$ & .08 \\
mRS $>2$ & $15(50.0 \%)$ & $5(21.7 \%)$ & \\
\hline a 2 & & &
\end{tabular}

${ }^{a}$ For comparison between IVT and IES.

Table 3: Multivariable analysis of favorable clinical outcome at 3 months

\begin{tabular}{lcccc}
\hline & OR $(95 \% \mathrm{CI})^{\mathrm{a}}$ & $\begin{array}{c}\boldsymbol{P} \\
\text { Value }^{\mathrm{b}}\end{array}$ & $\begin{array}{c}\text { adjOR } \\
(95 \% \mathrm{CI})^{\mathrm{a}}\end{array}$ & $\begin{array}{c}\boldsymbol{P} \\
\text { Value }^{\mathrm{b}}\end{array}$ \\
\hline IES & $2.99(0.86-12.3)$ & .08 & $11.9(1.6-89.1)$ & .02 \\
Initial NIHSS $<15$ & $0.78(0.67-0.90)$ & .001 & $12.5(1.8-87.0)$ & .01 \\
MRI ASPECTS $>7$ & $3.2(0.90-11.3)$ & .007 & $4.9(0.8-31.0)$ & .09 \\
Diabetes & $0.12(0.02-0.65)$ & .01 & $0.13(0.01-1.7)$ & 10 \\
Time to treatment & $1.00(0.99-1.02)$ & .63 & - & - \\
Dyslipidemia & $0.45(0.14-1.49)$ & .19 & - & - \\
Sex (male) & $0.88(0.28-2.80)$ & .83 & - & - \\
Age & $0.98(0.93-1.03)$ & .45 & - & - \\
Current smoker & $0.75(0.23-2.43)$ & .64 & - & - \\
Hypertension & $0.49(0.15-1.62)$ & .25 & - & - \\
\hline
\end{tabular}

Note:- OR indicates odds ratio; adjOR, adjusted odds ratio.

a OR and adjOR values comparing the 3-month clinical outcome between IVT and IES, after adjustment for confounding factors using an exact logistic regression.

${ }^{\mathrm{b}} \mathrm{P}$ for comparison between IVT and IES ( $\chi^{2}$ test or Fisher exact test).

not possible to assess the NIHSS score at 24 hours because a prolonged sedation was necessary ( $3 / 32$ belonged to the IVT group and $3 / 24$ to the IES group). At 3 months, $15 / 30$ patients (50\%) in the IVT group and $17 / 22(77 \%)$ in the IES group had an mRS score between 0 and 2 . Within the IES group, 10/15 patients with mechanical thrombectomies (67\%) and $7 / 7$ with IVT alone (100\%) had a favorable outcome (Fig 1).

As expected, a baseline NIHSS score of $<15$ was associated with a favorable outcome. After adjustment for this NIHSS score, IES was highly associated with a favorable outcome at 3 months (adjusted odds ratio $=11.9 ; 95 \% \mathrm{CI}, 1.6-89.1 ; P<.02$ ) (Table 3).

\section{Treatment Complications}

Twelve asymptomatic intracranial hemorrhages (parenchymal hematoma type 1) were observed on imaging at 24 hours. Seven patients were in the IVT group, and 5 , in the IES group. No symptomatic hemorrhages were observed.

\section{DISCUSSION}

The results of this observational study suggested that our therapeutic strategy (IES) based on rescue thrombectomy after initial IVT failure provided better clinical benefits for the patients in comparison with standard IVT alone. As expected, these favorable results were certainly due to higher recanalization rates (94\%). Furthermore, this strategy did not induce additional adverse events.

Previous results of IVT studies reported that MCA recanalization could be achieved in one-third of patients (between 32\% and
$35 \%$ ), with subsequent favorable clinical outcome at 3 months. ${ }^{2,3}$ Recently, it has been demonstrated that this recanalization rate can be significantly improved by using a combined IV-IA approach $^{6,9,11,16}$ and different mechanical devices in cases of thrombectomy. ${ }^{9,11,17,18}$ However, results in terms of clinical benefit at 3 months are mixed. On the basis of these observations and our preliminary protocol evaluation, the management of acute ischemic stroke in our institution was reviewed by our multidisciplinary stroke team in September 2009 and a compromise between IVT alone and combined IV-endovascular therapy was chosen. An IES by using mechanical thrombectomy (Solitaire FR) as a rescue therapy only in cases of IVT failure was systematically introduced for MCA occlusions.

In our center, the careful assessment of the acute ischemic lesions represents an important point in the decision to treat. Although parenchymal extension of the ischemic lesion by using the CT-ASPECTS was described as an alternative approach to the rule of one-third of the cerebral hemisphere, the DWI sequence is more objective in detecting ischemic lesions with a clearer contrast than CT. ${ }^{14}$ Thus, the DWI-ASPECTS applied was systematically assessed before the IVT decision, and patients having a predictive score of death (DWI-ASPECTS, $\leq 4$ ) were not treated and were excluded from the study. ${ }^{14}$ In addition, a DWI-ASPECTS of $>7$ has been shown to be a good prognostic factor for patients. ${ }^{14,19}$ This was also observed in our setting, though it did not reach statistical significance, possibly due to a suboptimal sample size. Our findings also confirmed that the initial clinical severity on presentation is correlated to the clinical prognosis. As shown before, ${ }^{20,21}$ the initial NIHSS score was identified as an independent predictive factor for favorable clinical outcome at 3 months.

Previous studies of acute MCA occlusions reported a favorable clinical outcome at 3 months in $41 \%$ and $63 \%$ of patients by using a combined IV-endovascular strategy, ${ }^{10,11}$ in $56 \%$ by using combined IV-IA thrombolysis, ${ }^{6}$ and in $40 \%$ and $53 \%$ by using IA thrombolysis alone. ${ }^{4,5}$ Comparatively, patients included in our IES group presented with high rates of favorable outcome at 3 months $(77 \%)$. This was also the case in patients who underwent only mechanical thrombectomy (67\%). The implementation of thrombectomy after initial IVT failure markedly improved the favorable outcome rate at 3 months, most particularly after adjustment for the initial clinical severity (NIHSS) between the 2 groups, with statistical significance. Stroke severity at admission was identified as the most important predictive factor of clinical outcome. Given that patients included in the IES group had higher initial NIHSS scores, a causal relationship between IES and 3-month favorable outcome is very likely, though our study was not randomized. Beyond the beneficial effect of the IES, our IVT control group also presented with high rates of favorable outcome at 3 months $(50 \%)$ in comparison with previous reports, which ranged from $35 \%$ to $49 \%$ in the MCA location. ${ }^{5,20}$

All the series presented above suggested that an early arterial recanalization is a significant end point in acute stroke. However it should not be considered alone because the favorable outcome in our setting could not be explained by the time from symptom onset to IVT, which was longer than that in other studies. We believe this is secondary to a combination of the geographic dif- 
ficulty presented by our rural catchment area and the relatively long door-to-needle time in this study. ${ }^{5,6,9,11,16,20}$ The strict patient-selection criteria based on the location-weighted imaging system (ASPECTS) systematically used in our center for all patients might be another additional explanation. It may be that the parenchymal extension of the ischemic lesion could have been underestimated on CT in most of the previous studies, increasing worse outcome and hemorrhagic complication rates.

Another potential factor influencing the favorable clinical outcome in our setting could be related to the use of the Solitaire FR stent device, which achieved an MCA recanalization rate of $94 \%$. The stent was easily navigated to the occlusion point, and its deployment across the thrombus was obtained at first attempt in all cases. ${ }^{22}$ No symptomatic complications (ie, intracranial hemorrhage) were observed in the IES group, suggesting a good safety profile of this stent retriever in the MCA territory. Recent reports have also demonstrated equally encouraging results in terms of the efficacy (ie, recanalization) and safety with this device. ${ }^{22-24}$

The major limitation of this study was the absence of patient randomization between IVT and IES groups, which may have biased our results. However, these 2 groups consisted of consecutive cases comparable in regard to classic prognostic factors, and the results were based on a multivariable analysis with an adjustment on confounding factors. Given the high significant association between IES and favorable clinical outcome at 3 months, it is unlikely that any residual confounding factors would alter our findings dramatically. The IVT procedure and the inclusion/exclusion criteria did not change during the study period. Our stroke center optimized with time the rapid referral of patients with stroke, which could explain the lower time from symptom onset to treatment in the second study period. However, this difference was accounted for in the analysis and was not associated with the study outcome.

\section{CONCLUSIONS}

Our results highlight the 24-hour and 3-month clinical benefit of an IV-endovascular strategy by using thrombectomy as a secondline therapy in patients presenting with an MCA occlusion within 4.5 hours. This strategy did not provide additional symptomatic complications. Arterial recanalization is still the most relevant goal, particularly when methodic selection of patients by using the DWI ASPECTS is performed. While the results of large multicenter trials that are evaluating the efficacy and safety of thrombectomy as a first-line therapy are pending, our strategy could represent an interesting alternative in the management of acute MCA occlusions.

\section{REFERENCES}

1. Bluhmki E, Chamorro A, Machnig T, et al. Stroke treatment with alteplase given $3.0-4.5 \mathrm{~h}$ after onset of acute ischaemic stroke (ECASS III): additional outcomes and subgroup analysis of a randomized controlled trial. Lancet Neurol 2009;8:1095-102

2. del Zoppo GJ, Poeck K, Pessin MS, et al. Recombinant tissue plasminogen activator in acute thrombotic and embolic stroke. Ann Neurol 1992;32:78-86

3. Bhatia $\mathrm{R}, \mathrm{Hill} \mathrm{MD}$, Shobha N, et al. Low rates of acute recanalization with intravenous recombinant tissue plasminogen activator in ischemic stroke: real-world experience and a call for action. Stroke 2010;41:2254-58
4. Furlan A, Higashida R, Wechsler L, et al. Intra-arterial prourokinase for acute ischemic stroke: the PROACT II study: a randomized controlled trial—Prolyse in Acute Cerebral Thromboembolism. JAMA 1999;282:2003-11

5. Mattle HP, Arnold M, Georgiadis D, et al. Comparison of intraarterial and intravenous thrombolysis for ischemic stroke with hyperdense middle cerebral artery sign. Stroke 2008;39:379-83

6. Sekoranja L, Loulidi J, Yilmaz H, et al. Intravenous versus combined (intravenous and intra-arterial) thrombolysis in acute ischemic stroke: a transcranial color-coded duplex sonography-guided pilot study. Stroke 2006;37:1805-09

7. Rubiera M, Ribo M, Pagola J, et al. Bridging intravenous-intra-arterial rescue strategy increases recanalization and the likelihood of a good outcome in a nonresponder intravenous tissue plasminogen activator-treated patients: a case-control study. Stroke 2011;42: 993-97

8. Becktepe JS, You SJ, Berkefeld J, et al. Clinical outcome after mechanical recanalization as mono- or adjunctive therapy in acute stroke: importance of time to recanalization. Cerebrovasc Dis 2011; 32:211-18

9. Mazighi M, Serfaty JM, Labreuche J, et al. Comparison of intravenous alteplase with a combined intravenous-endovascular approach in patients with stroke and confirmed arterial occlusion (RECANALISE) study: a prospective cohort study. Lancet Neurol 2009;8:802-09

10. Shi ZS, Loh Y, Walker G, et al. Endovascular thrombectomy for acute ischemic stroke in failed intravenous tissue plasminogen activator versus non-intravenous tissue plasminogen activator patients: revascularization and outcomes stratified by the site of arterial occlusions. Stroke 2010;41:1185-92

11. Sugiura S, Iwaisako K, Toyota S, et al. Simultaneous treatment with intravenous recombinant tissue plasminogen activator and endovascular therapy for acute ischemic stroke within 3 hours of onset. AJNR Am J Neuroradiol 2008;29:1061-66

12. Tissue plasminogen activator for acute ischemic stroke: the National Institute of Neurological Disorders and Stroke rt-PA Stroke Study Group. N Engl J Med 1995;333:1581-87

13. Costalat V, Machi P, Lobotesis K, et al. Rescue, combined, and standalone thrombectomy in the management of large vessel occlusion stroke using the Solitaire device: a prospective 50-patient singlecenter study-timing, safety, and efficacy. Stroke 2011;42:1929-35

14. Nezu T, Koga M, Kimura K, et al. Pretreatment ASPECTS on DWI predicts 3-month outcome following rt-PA: SAMURAI rt-PA registry. Neurology 2010;75:555-61

15. Higashida RT, Furlan AJ, Roberts H, et al, Technology Assessment Committee of the American Society of Interventional and Therapeutic Neuroradiology; Technology Assessment Committee of the Society of Interventional Radiology. Trial design and reporting standards for intra-arterial cerebral thrombolysis for acute ischemic stroke. Stroke 2003;34:109-37

16. The IMS II Trial Investigators. The interventional management of stroke (IMS) II study. Stroke 2007;38:2127-35

17. Nogueira RG, Liebeskind DS, Sung G, et al, for the Multi MERCI Writing Committee. Predictors of good clinical outcomes, mortality, and successful revascularization in patients with acute ischemic stroke undergoing thrombectomy: pooled analysis of the Mechanical Embolus Removal in Cerebral Ischemia (MERCI) and Multi MERCI trials. Stroke 2009;40:3777-83

18. Penumbra Pivotal Stroke Trial Investigators. The Penumbra Pivotal Stroke Trial: safety and effectiveness of a new generation of mechanical devices for clot removal in intracranial large vessel occlusive disease. Stroke 2009;40:2761-68

19. Hill MD, Rowley HA, Adler F, et al. Selection of acute ischemic stroke patients for intra-arterial thrombolysis with pro-urokinase by using ASPECTS. Stroke 2003;34:1925-31

20. Molina CA, Alexandrov AV, Demchuk AM, et al. Improving the predictive accuracy of recanalization on stroke outcome in patients treated with tissue plasminogen activator. Stroke 2004;35:151-56 
21. Machumpurath B, David SM, Yan B. Rapid neurological recovery after intravenous tissue plasminogen activator in stroke: prognostic factors and outcome. Cerebrovasc Dis 2011;31:278-83

22. Machi P, Costalat V, Lobotesis K, et al. Solitaire FR thrombectomy system: immediate results in $\mathbf{5 6}$ consecutive acute ischemic stroke patients. J Neurointerv Surg 2012;4:62-66
23. Castano C, Dorado L, Guerrero C, et al. Mechanical thrombectomy with the Solitaire AB device in large artery occlusions of the anterior circulation: a pilot study. Stroke 2010;41:1836-40

24. Roth C, Papanagiotou P, Behnke S, et al. Stent-assisted mechanical recanalization for treatment of acute intracerebral artery occlusions. Stroke 2010;41:2559-67 